Ver sat i l i ty of chi tosan/BnNPV bacmid DNA nanocompl ex as transf ect i on reagent of recombi nant prot ei $n$ expressi on i $n$ si I kworm I arvae

\begin{tabular}{|c|c|}
\hline 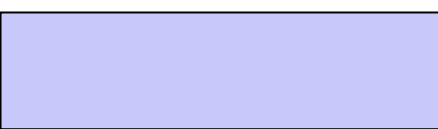 & $\begin{array}{l}\text { Kat o Tat suya, Ar ai Sho, I chi kawa H rono, Park } \\
\text { Enoch Y. }\end{array}$ \\
\hline $\begin{array}{l}\text { j our nal or } \\
\text { publ i cat i on title }\end{array}$ & Bi ot echnol ogy Letters \\
\hline vol une & 38 \\
\hline nunber & 9 \\
\hline page $r$ ange & 1449-1457 \\
\hline year & 2016-06- 07 \\
\hline 出版者 & Spr i nger Net her I ands \\
\hline 権利 & $\begin{array}{l}\text { ( C) Spr i nger Sci ence+Busi ness Medi a Dor dr echt } \\
2016\end{array}$ \\
\hline URL & ht t p: //hdl . handl e. net /10297/10286 \\
\hline
\end{tabular}




\title{
1 Versatility of chitosan/BmNPV bacmid DNA nanocomplex as \\ 2 transfection reagent of recombinant protein expression in silkworm \\ 3 larvae
}

\author{
4 Tatsuya Kato $\cdot$ Sho Arai $\cdot$ Hirono Ichikawa $\cdot$ Enoch Y. Park* \\ 5 \\ 6 E-mail: \\ 7 kato.tatsuya@shizuoka.ac.jp (TK) \\ 8 revn_ed@yahoo.co.jp (SA) \\ 9 itikawahirono@gmail.com (HI) \\ 10 kenpi901@yahoo.co.jp (KT) \\ 11 park.enoch@shizuoka.ac.jp (EYP)
}

\footnotetext{
* Tatsuya Kato $\cdot$ Enoch Y. Park (凶)

Laboratory of Biotechnology, Green Chemistry Research Division, Research Institute of Green Science and Technology, Shizuoka University, 836 Ohya Suruga-ku, Shizuoka, 422-8529, Japan

Laboratory of Biotechnology, Department of Bioscience, Graduate School of Science and Technology, Shizuoka University, 836 Ohya, Suruga-ku, Shizuoka, 422-8529, Japan

e-mail: park.enoch@shizuoka.ac.jp , Phone \& Fax: +81-54-238-4887
}

Tatsuya Kato $\cdot$ Sho Arai $\cdot$ Hirono Ichikawa $\cdot$ Enoch Y. Park

Laboratory of Biotechnology, Department of Applied Biological Chemistry, Faculty of Agriculture, Shizuoka University, 836 Ohya, Suruga-ku, Shizuoka, 422-8529, Japan 


\section{Abstract}

13 Objective The feasibility of chitosan as an alternative transfection reagent candidate

14 was examined for protein expression in Bm5 cells and silkworm larvae using

15 recombinant BmNPV bacmid DNA.

16 Results Chitosan 100 and recombinant Bombyx mori nucleopolyhedrovirus

17 (BmNPV) bacmid DNA, in amino group/phosphate group (N/P) ratios of $0.1-10$, were

18 used for formation of chitosan/DNA nanocomplexes. The chitosan/BmNPV bacmid

19 DNA nanocomplexes showed higher specific activity of

$20 \mathrm{GFP}_{\mathrm{uv}}-\beta 1,3-N$-acetylglucosaminyltransferase 2 ( $\left.\beta 3 \mathrm{GnT} 2\right)$ fusion protein (GGT2)

21 expressed in silkworm larvae than DMRIE-C, a conventional silkworm transfection

22 reagent. In particular, the composition of chitosan and BmNPV bacmid DNA

23 nanocomplexes formed by an N/P ratio of 8 or 10, respectively, showed the highest

24 specific activity of $\beta 3 \mathrm{GnT} 2$ in the silkworm larvae hemolymph. In addition, 3 different

25 proteins were expressed in silkworm larvae to the same extent using chitosan as that

26 using DMRIE-C.

27 Conclusion This is the first finding that chitosan/BmNPV bacmid DNA

28 nanocomplexes can rival the performance of commercially available transfection

29 reagents for the expression of recombinant proteins in Bm5 cells and silkworm larvae.

30 Keywords Chitosan $\cdot$ Silkworm $\cdot$ BmNPV bacmid $\cdot$ Recombinant protein

$31 \cdot$ Nanocomplex $\cdot \beta 1,3-N$-Acetylglucosaminyltransferase $\cdot$ Chitosan-DNA

32 nanocomplexes 


\section{Introduction}

34 Chitosan is a cationic, water-soluble, linear polymer extracted from crustacean shells that easily forms nanocomplexes with drugs, nucleic acids and proteins through electrostatic interactions. This interaction between chitosan and biomolecules leads to the production of biomaterials for a variety of biomedical applications, such as drug and gene delivery and tissue engineering (Garcia-Fuentes and Alonso, 2012). The biocompatibility and biodegradability properties of chitosan make it suitable for most in vivo medical applications. In particular, chitosan can be digested by the lysozymes $\mathrm{N}$-acetylglucosaminidase and chitotriosidase, which exist in human mucosa and various physiological fluids (Gorzelanny et al., 2010; Kean et al., 2010).

Chitosan also can be utilized for gene delivery as a non-viral delivery system instead of cationic polymers and liposomes. Chitosan has protonated amine groups, the positive charge of which can electrostatically interact with DNA and RNA, allowing chitosan to be delivered into cells as nanocomplexes. Chitosan-nucleic acid nanocomplexes can be prepared by coacervation at low cost. In several reports, chitosan was modified by thiamine pyrophosphate, polyethylenimine and hyaluronic acid to enhance its in vitro transfection efficiency compared with that of commercially available transfection reagents (de la Fuente et al., 2008; Jian et al., 2009; Rojanarata et al., 2008; Steg et al., 2011; Tripathi et al., 2012). In the transfection of nucleic acids to cultured cells and tissues using chitosan, plasmid vectors, siRNA and shRNA have normally been used to express recombinant proteins and suppress the expression of specific genes.

In this study, chitosan was used instead of commercially available transfection reagents for recombinant protein expression in Bombyx mori ovary (Bm5) cells and silkworm larvae. To express recombinant proteins in Bm5 cells and silkworm larvae, 
recombinant B. mori nucleopolyhedrovirus (BmNPV) bacmid DNA was also used (Motohashi et al., 2005). The size of the BmNPV bacmid is approximately $130 \mathrm{kbp}$, which is much larger than that of plasmid vectors; the transfection of large DNA such as

60 the BmNPV bacmid has not previously been conducted. To confirm the transfection 61 efficiency, GFP $\mathrm{uv}_{-} \beta 1,3-N$-acetylglucosaminyltransferase 2 ( $\left.\beta 3 \mathrm{GnT} 2\right)$ fusion protein 62 (GGT2) was expressed in Bm5 cells and silkworm larvae (Park et al., 2007) using the chitosan/BmNPV bacmid DNA nanocomplexes. To demonstrate the versatility of these

64 chitosan-based nanocomplexes, rat $\alpha 2,6$-sialyltransferase (ST6), hemagglutinin (HA) from an influenza A virus, and the Neospora caninum surface protein (NcSRS2) were expressed using the chitosan/BmNPV bacmid DNA nanocomplexes. The efficiency and cost of this expression method compared with a commercial transfection reagent was 68 also discussed.

\section{Materials and methods}

Construction of recombinant BmNPV bacmids

72 Recombinant BmNPV bacmids containing the GGT2 gene (Kato et al., 2004),

73 recombinant BmNPV bacmids for the expression of rat ST6 (Ogata et al., 2009) and

74 NcSRS2 (Otsuki et al. 2013) have been constructed in previous studies. The construction of recombinant BmNPV bacmids containing the HA gene from an influenza A virus is briefly described below. A plasmid vector containing the HA gene

77 from influenza A H5N8 (A/duck/NY/191255-59/02) was purchased from Sino

78 Biological Inc. (Beijing, China). The FLAG-tagged HA gene lacking the sequence

79 coding its signal peptide was amplified by PCR using HA-1st-F 
’) and HA-1st-R (5'-GGGGTACCTTAGATACAAATCCTACATTGGAGGGA-3’).

Using the amplified HA gene as a template and primers (HA-2nd-F:

AAGTCTGACCAGATTGACTACAAGGATGACG-3’, HA-1st-R), the FLAG-tagged whole HA gene was amplified by PCR. The amplified, FLAG-tagged whole HA gene was inserted into the pFastBac1 vector and the constructed recombinant vector was transformed into Escherichia coli BmDH10bac CP-Chi' (Park et al., 2008). Recombinant BmNPV bacmids containing the HA gene were extracted from a white colony through blue-white selection by the PureLink HiPure Plasmid DNA Purification Kit (Life Technologies Japan, Tokyo, Japan)

Preparation of the chitosan/BmNPV bacmid DNA nanocomplexes

Chitosan 10, 100, and 500 were purchased from Wako Pure Chemical Ind. Ltd. (Osaka, Japan). Chitosan was dissolved with $50 \mathrm{mM}$ acetate, and the $\mathrm{pH}$ was adjusted to 5.5 using $0.1 \mathrm{M} \mathrm{NaOH}$. Recombinant BmNPV bacmid DNA was dissolved with 2-( $N$-morpholino)ethanesulfonic acid (MES) buffer (pH 6.5). In brief, 0.1\% (w/v)

96 Chitosan and each amount of recombinant BmNPV bacmid DNA were prepared with MES buffer (pH 6.5), heated separately to $50-55^{\circ} \mathrm{C}$, and quickly mixed with each other according to amino group/phosphate group (N/P) ratio. The N/P ratios were calculated by the formula described below.

$$
\mathrm{N} / \mathrm{P} \text { ratio }=\frac{\text { Chitosan }(g) \times 0.8}{D N A(g)} \times \frac{330(\text { average molecular weight of each nucleotide })}{161(\text { molecular weight of deacetylated GlcNAc) }}
$$


103 The chitosan 100 used in this study was approximately $80 \%$ deacetylated chitin. This

104 mixture was incubated for $1 \mathrm{~h}$ at room temperature and subsequently used as the

105 chitosan/BmNPV bacmid DNA nanocomplexes. Size and zeta potential of these

106 nanocomplexes were analyzed by dynamic light scattering (DLS) analysis (Zetasizer

107 Nano ZS, Malvern Instruments, Worcestershire, UK).

108

109 Cultivation of Bm5 cells and rearing silkworm larvae

110 The Bm5 cells were cultivated at $27^{\circ} \mathrm{C}$ in Sf-900II medium (Life Technologies Japan)

111 supplemented with 10\% fetal bovine serum (FBS, Sigma Aldrich Japan, Tokyo, Japan)

112 and 100-fold-diluted Antibiotic-Antimycotic (Life technologies Japan). Fourth instar

113 silkworms were purchased from Ehime Sansyu (Ehime, Japan) and reared at $26^{\circ} \mathrm{C}$ on an

114 artificial diet (Silkmate 2S, Nosan, Yokohama, Japan).

115 Agarose gel electrophoresis analysis of chitosan/BmNPV bacmid DNA nanocomplexes

116 Chitosan/BmNPV bacmid DNA nanocomplexes containing $10 \mu \mathrm{g}$ of BmNPV bacmid

117 DNA were treated with $2 \mathrm{U}$ of DNase (RT-grade, Wako) for $15 \mathrm{~min}$ at $37^{\circ} \mathrm{C}$, and the

118 reaction was stopped by adding the stop solution provided. The reaction mixture was

119 analyzed using $1 \%$ agarose gel electrophoresis.

120 Transfection and injection of chitosan/BmNPV bacmid DNA nanocomplexes into Bm5

121 cells and silkworm larvae

122 Using a 6-well plate, $8 \times 10^{5} \mathrm{Bm} 5$ cells were cultivated in each well. After removal of

123 the culture medium, Cellfectin II (Life Technologies Japan)-BmNPV bacmid DNA 
125 bacmid DNA: 2.5, 5 and $10 \mu \mathrm{g}$ ) were added into each well, and the plates were

126 incubated for $5 \mathrm{~h}$. Two milliliter of fresh culture medium supplemented with $10 \%$ FBS

127 was added into each well following the removal of the Cellfectin II-BmNPV bacmid

128 DNA complexes. Transfected Bm5 cells were cultivated for $7 \mathrm{~d}$. In the case of

129 chitosan/BmNPV bacmid DNA nanocomplexes, $2 \mathrm{ml}$ of fresh culture medium

130 supplemented with $10 \%$ FBS was added into each well, the nanocomplexes were not

131 removed, and the cells were cultivated for $7 \mathrm{~d}$. Cells and culture supernatants were

132 collected and used for further experiments.

133 DMRIE-C (a 1:1 (M/M) liposome formulation of the cationic lipid DMRIE (1,2-

134 dimyristyloxy-propyl-3-dimethyl-hydroxy ethyl ammonium bromide) and cholesterol in 135 membrane filtered water, Life Technologies Japan)-BmNPV bacmid DNA complexes

136 or chitosan/BmNPV bacmid DNA nanocomplexes (BmNPV bacmid DNA: $5 \mu \mathrm{g}$ ) were

137 injected into fifth instar silkworm larvae, and the injected larvae were reared for $7 \mathrm{~d}$ on

138 an artificial diet (Silkmate S2, Nohsan Corporation, Yokohama, Japan). Hemolymph

139 and body fat were collected from the injected silkworm larvae and used for further

140 experiments.

141 Culture supernatants and hemolymph were centrifuged at $10,000 \times \mathrm{g}$, and the

142 resulting supernatant was used as a sample. Cells and body fat were suspended with

143 PBS (pH 7.4) and disrupted by sonication. The homogenate was centrifuged at 10,000 $\times$

$144 \mathrm{~g}$, and its supernatant was used as a sample.

$145 \quad \beta 3 \mathrm{GnT}$ assay and protein concentration measurement 
146 The $\beta 3 \mathrm{GnT}$ assay was conducted according to a previously reported method (Kato et al.,

147 2004). The protein concentration of the silkworm hemolymph was determined using a

148 BCA protein assay kit (Thermo Fisher Scientific K.K., Yokohama, Japan).

149 Detection of expressed proteins by fluorescence microscopy, SDS-PAGE and western 150 blot

151 Transfected Bm5 cells were immobilized on a glass slide coated with aminosilane

152 (Matsunami Glass, Osaka, Japan). Cells were fixed with $10 \%$ formalin and washed by

153 PBS 4 times. Fluorescence was observed using a confocal laser scanning microscope

154 (LSM700, Carl Zeiss Japan, Tokyo, Japan).

155 Expression of recombinant proteins was confirmed by sodium dodecyl

156 sulfate-polyacrylamide gel electrophoresis (SDS-PAGE) using $10 \%$ or $12 \%$ acrylamide

157 gels. In the case of GGT2, the samples were mixed with sample buffer (Aoki et al.,

158 1996) without boiling and processed with SDS-PAGE. GGT2 was detected using

159 Molecular Imager FX (Bio-Rad, Hercules, CA, USA). Other recombinant proteins were

160 detected by western blot. After SDS-PAGE, proteins were electrotransferred onto a

161 polyvinylidene fluoride (PVDF) membrane using the Mini Trans-Blot Electrophoretic

162 Transfer Cell (Bio-Rad). After blocking in 5\% skimmed milk in TBS containing 0.1\%

163 Tween 20 (TBST), the membrane was incubated for $1 \mathrm{~h}$ in 1:10000 mouse

164 anti-DDDDK antibody (Medical \& Biological Laboratories, Nagoya, Japan). The

165 membrane was washed with TBST and incubated for $1 \mathrm{~h}$ in 1:20000 anti-mouse $\operatorname{IgG}$

166 antibody labeled with horseradish peroxidase (GE Healthcare Japan, Tokyo, Japan).

167 Detection was performed with Immobilon Western Chemiluminescent HRP Substrate 


\section{$171 \quad$ Results}

172 Preparation of the chitosan/BmNPV bacmid DNA nanocomplexes

173 Chitosan is cationic because of its amine groups. In this study, the ratio of acetylated

174 amine groups had a great effect on chitosan solubility. The chitosan 10, 100 and 500

175 used were deacetylated by more than $80 \%$, according to the commercial information. In

176 a preliminary experiment, pH 6.5 MES buffer was shown to be favorable for forming

177 chitosan/BmNPV bacmid DNA nanocomplexes using Chitosan 100. Optimal pH for the

178 chitosan/DNA nanocomplexes to obtain the adequate balance between DNA association

179 and dissociation is slightly lower than 7 (Mao et al., 2010). Therefore, we used pH 6.5

180 MES buffer for the preparation of the chitosan/BmNPV bacmid DNA nanocomplexes.

181 Chitosan 100 was used to prepare the chitosan/BmNPV bacmid DNA

182 nanocomplexes. Different types of these nanocomplexes were prepared using various

183 N/P ratios ranging from $0.1-10$; their resulting sizes and zeta potentials are shown in

184 Fig. 1. As the N/P ratio increased, the nanocomplexes increased in size from

185 approximately $70 \mathrm{~nm}$ to $120 \mathrm{~nm}$. When the chitosan formed nanocomplexes with

186 sodium sulfate in the absence of DNA they were approximately $80 \mathrm{~nm}$ in diameter. Fig.

1871 indicates that at N/P ratios greater than 2, Chitosan 100 can form larger nanocomplexes with BmNPV bacmid DNA than that without DNA. In a previous study, 
189 large aggregates were found when chitosan and plasmid DNA were mixed at an N/P

190 ratio of approximately 1 (Mao et al., 2001), but no aggregation was detected in this

191 study; these nanocomplexes were smaller than $130 \mathrm{~nm}$ for all N/P ratios (Fig. 1).

192 Chitosan/BmNPV bacmid DNA nanocomplexes prepared at N/P ratios ranging from

193 0.1-10 were analyzed using agarose gel electrophoresis (Fig. 2a). The size of BmNPV

194 bacmid DNA is approximately $130 \mathrm{kbp}$, and it is observed above $10 \mathrm{kbp}$ in an agarose

195 gel. At N/P ratios of 0.1 and 0.3, some of the BmNPV bacmid DNA was observed

196 around the wells and the rest was observed at the same location as naked BmNPV

197 bacmid DNA, indicating that chitosan/BmNPV bacmid DNA partially formed at these

198 N/P ratios. At N/P ratios greater than 0.5, all of the BmNPV bacmid DNA formed

199 nanocomplexes with chitosan. Next, DNase treatment of chitosan/BmNPV bacmid

200 DNA was performed to confirm the protection of the BmNPV bacmid DNA by chitosan

201 in these nanocomplexes (Fig. 2b). BmNPV bacmid DNA was not observed in the

202 nanocomplexes at N/P ratios of $0.5,0.8$, and 1 after DNase treatment, but it was

203 observed in nanocomplexes at N/P ratios greater than 4 even though DNase treatment

204 was performed. This result indicates that chitosan/BmNPV bacmid DNA

205 nanocomplexes formed incompletely between N/P ratios of 0.5 and 1 because the

206 amount of chitosan was limited; at N/P ratio greater than 4, nanocomplexes completely

207 coated with chitosan formed, which completely protected BmNPV bacmid DNA from

208 digestion by DNase.

209 Expression of recombinant proteins in Bm5 cells using chitosan/BmNPV bacmid DNA

210 nanocomplexes 
211 Chitosan/BmNPV bacmid DNA nanocomplexes were prepared at N/P ratios of 4 and 6

212 using chitosan 100 and BmNPV bacmid DNAs containing the GGT2 fusion gene, and

213 these nanocomplexes were transfected into Bm5 cells. Green fluorescence was not only

214 observed in Bm5 cells transfected with Cellfectin II-BmNPV bacmid DNA complexes

215 but also in Bm5 cells transfected with chitosan/BmNPV bacmid DNA nanocomplexes

216 (Fig. 3a). However, Bm5 cell transfection only with bacmid DNA did not yield any

217 green fluorescence. These results indicate that chitosan 100 can function at the same

218 level as Cellfectin II as a Bm5 cell transfection reagent. GGT2 expression in Bm5 cell

219 was used to assess BmNPV bacmid DNA doses between 2.5 and $10 \mu \mathrm{g}$ at N/P ratios 4

220 and 6. SDS-PAGE analysis of intracellular GGT2 expression was higher using

221 nanocomplexes at an N/P ratio of 6 than that at an N/P ratio of 4 (Fig. 3b). In all

222 conditions at an N/P ratio of 6,5 $5 \mathrm{~g}$ of BmNPV bacmid DNA in each well of a 6-well

223 plate $\left(8 \times 10^{5}\right.$ cells) yielded the highest expression of GGT2 (Fig. 3b). These results

224 indicate that chitosan 100 can be used for protein expression using BmNPV bacmid in

225 Bm5 cells as a transfection reagent instead of Cellfectin II.

226 Expression of recombinant proteins in silkworm larvae using chitosan/BmNPV bacmid

227 DNA nanocomplexes

228 GGT2 in silkworm larvae was expressed using the chitosan/BmNPV bacmid DNA

229 nanocomplexes. DMRIE-C was used as a transfection reagent for silkworm larvae and

230 pupae. In the case of the DMRIE-C reagent, 8 out of 10 silkworm larvae exhibited green

231 fluorescence at $7 \mathrm{~d}$ after injection of the DMRIE-C-BmNPV bacmid DNA complexes

232 (Fig. 4a). Compared to DMRIE-C, almost the same transfection efficiency (5-8 out of

23310 silkworm larvae) was achieved by injection of chitosan/BmNPV bacmid DNA 
234 nanocomplexes between N/P ratios of 2 and 8 (Fig. 4a). Injection of only naked

235 recombinant BmNPV bacmid DNA did not allow GGT2 to be expressed in silkworm

236 larvae. GGT2 expression was observed in the hemolymph from silkworm larvae

237 injected with DMRIE-C-BmNPV bacmid DNA complexes, chitosan/BmNPV bacmid

238 DNA nanocomplexes between N/P ratios 2 and 8, and mock (Fig. 4b). Specific $\beta 3 \mathrm{GnT}$

239 activity observed in the hemolymph at each N/P ratio was higher than that observed

240 after using DMRIE-C (Fig. 5). In particular, the silkworm larvae injected with

241 chitosan/BmNPV bacmid DNA nanocomplexes with N/P ratios of 8 or 10 exhibited the

242 highest specific activity of $\beta 3 \mathrm{GnT}$ in the hemolymph. These results indicate that

243 chitosan 100 can be also used for protein expression using BmNPV bacmid in silkworm

244 larvae as a transfection reagent instead of DMRIE-C.

245 Feasibility of chitosan/BmNPV bacmid DNA nanocomplexes as transfection agent for

246 expression of recombinant proteins in silkworm larvae

247 The expression of rat ST6, HA from the influenza A H5N8 virus and NcSRS2 was

248 investigated using chitosan/BmNPV bacmid DNA nanocomplexes in silkworm larvae.

249 Rat ST6 and NcSRS2 from N. caninum were expressed in the hemolymph because the

250 transmembrane domain of each protein was deleted (Ogata et al., 2009; Otsuki et al.,

251 2013). All three recombinant proteins were expressed in the silkworm larvae

252 hemolymph (Fig. 6). Expressed HA of the influenza A H5N8 virus was observed in the

253 hemolymph at approximately $50 \mathrm{kDa}$ even when this HA had its own transmembrane

254 domain. This result suggested that the HA expressed in the hemolymph was observed as

255 HA1 because the estimated molecular weight of this HA in Fig. 6 is approximately 50 
$256 \mathrm{kDa}$. These results indicate that chitosan can be used as an inexpensive substituent for

257 DMRIE-C for the expression of any recombinant protein in silkworms.

258 GGT2 was also expressed in silkworm larvae using chitosan 10 and 500 instead of 259 chitosan 100. Chitosan 10 has the smallest molecular weight among these three types of 260 chitosan, while Chitosan 500 has the largest. In addition, chitosan 10 and 500 mediated

261 the expression of GGT2 in the hemolymph to the same extent as did chitosan 100 (data 262 not shown).

\section{Discussion}

264 The transfection efficiency of DNA into cells by chitosan depends on various

265 parameters, including the molecular weight and deacetylation degree of chitosan, the 266 N/P ratio of chitosan/DNA particles, the $\mathrm{pH}$, and additives, among others (Mao et al., 267 2010). In this study, at N/P ratios between 2 and 10, higher levels of GGT2 expression

268 in hemolymph were observed than those resulting from use of DMRIE-C. In the

269 BmNPV bacmid-silkworm expression system, BmNPV is produced and amplified,

270 leading to a systemic infection in the silkworm larvae when even the slightest amount of

271 BmNPV bacmid is introduced into silkworm larvae cells. This suggests that perfect

272 transfection conditions are not needed for the recombinant protein expression in

273 silkworm larvae. However, nanocomplexes composed of chitosan/BmNPV bacmid

274 DNA at N/P ratios of 8 or 10, respectively, showed the highest specific activity of

$275 \beta 3 \mathrm{GnT}$ in the silkworm larvae hemolymph. This result indicates that a high N/P ratio is

276 favorable for the expression of recombinant proteins in the BmNPV bacmid-silkworm

277 system. In general, higher molecular weight chitosan provides high transfection 278 efficiency at lower N/P ratio, but lower molecular weight chitosan requires higher N/P 
279 ratios to form chitosan/DNA nanocomplexes completely (Lavertu et al., 2006; Romøren

280 et al., 2003). To obtain high transfection efficiency with this chitosan-based formulation,

281 the optimization of N/P ratio and chitosan molecular weight is required (Mao et al.,

282 2010; Sato et al, 2001). The average molecular weight of the chitosan 100 used in this

283 study has not been informed from the manufacturer. However, from the result shown in

284 Fig. 4, it is possible that further optimization of the N/P ratio and chitosan molecular

285 weight will provide the highest expression of recombinant proteins in BmNPV

286 bacmid-silkworm system. In this case, it should be taken into consideration that the size

287 of BmNPV bacmid DNA is larger than that of plasmid DNA and siRNA, which have

288 been normally used for chitosan-based transfection.

289 In this study, recombinant proteins were expressed in Bm5 cells and silkworm

290 larvae using chitosan instead of the conventional transfection reagents Cellfectin II and

291 DMRIE-C. GGT2 expression in silkworm larvae hemolymph using chitosan 100 was

292 higher than that achieved by using DMRIE-C. Similar rat ST6 expression resulted from

293 the use of chitosan 100 and DMRIE-C. In addition, HA from the influenza A H5N8

294 virus and NcSRS2 from $N$. caninum exhibited comparable expression resulting from the

295 use of chitosan 100 and DMRIE-C. Conventionally, DMRIE-C, provided by Life

296 Technologies, is used to express recombinant proteins in silkworms by injecting

297 recombinant BmNPV bacmid DNA. In this case, $5 \mu$ l of DMRIE-C is needed to inject

298 recombinant bacmid DNA into a silkworm larva, and its cost for the recombinant

299 protein expression in a silkworm is 2.6 US\$. Using chitosan 100, $36 \mu \mathrm{g}$ of chitosan 100

300 is required for the recombinant protein expression in a silkworm, and its cost is $4.0 \times$

$30110^{-7}$ US\$. Chitosan 100 is much less expensive to use than DMRIE-C for the expression 
302 of recombinant proteins in silkworms and may lead to the cost-effective, large-scale

303 production of recombinant proteins in silkworms.

304 In conclusion, we developed a highly cost-effective transfection method using

305 chitosan 100. Chitosan 100 and recombinant BmNPV bacmid DNA easily formed

306 chitosan/BmNPV bacmid DNA nanocomplexes, which protected DNA from digestion

307 by DNase when prepared with an N/P ratio greater than 4 and an average size of $100 \mathrm{~nm}$.

308 Using these nanocomplexes as well as the commercial transfection reagent Cellfectin II,

309 several proteins were expressed successfully. In the case of GGT2, the composition of

310 chitosan and BmNPV bacmid DNA nanocomplexes formed by an N/P ratio of 8 or 10 ,

311 respectively, showed the highest specific activity of $\beta 3 \mathrm{GnT} 2$ in the silkworm larvae

312 hemolymph. In addition, recombinant proteins originating from a rat, influenza virus

313 and Neospora caninum surface protein were expressed in the silkworm larvae using

314 chitosan 100 to the same extent as expression resulting from the use of DMRIE-C. This

315 is the first demonstration of chitosan/BmNPV bacmid DNA nanocomplexes rivaling

316 silkworm protein expression achieved by commercially available transfection reagents,

317 and these results may lead to significantly reduced cost for recombinant protein

318 expression compared with the cost of conventional transfection methods.

\section{References}

320 Aoki T, Takahashi Y, Koch KS, Leffert, HL, Watabe, H (1996) Construction of a fusion

321 protein between protein A and green fluorescent protein and its application to

322 western blotting. FEBS Lett 384:193-197 
323 de la Fuente M, Seijo B, Alonso MJ (2008) Bioadhesive hyaluronic acid-chitosan

324 nanoparticles can transport genes across the ocular mucosa and transfect ocular

$325 \quad$ tissues. Gene Ther 15:668-676

326 Garcia-Fuentes M, Alonso MJ (2012) Chitosan-based drug nanocarriers: Where do we

327 stand? J. Control. Release 161:496-504

328 Gorzelanny C, Pöppelmann B, Pappelbaum K, Moershbacher BM, Schneider SW

329 (2010) Human macrophage activation triggered by chitotriosidases-mediated chitin

$330 \quad$ and chitosan degradation. Biomaterials 31:8556-8563

331 Jiang HL, Xu CX, Kim YK, Arote R, Jere D, Lim HT, Cho MH, Cho CS (2009) The

332 suppression of lung tumorigenesis by aerosol-delivered

333 folate-chitosan-graft-polyethylenimine/AKT1 ahRNA complexes through the AKT

$334 \quad$ signaling pathway. Biomaterials 30:5844-5852

335 Kean T, Thanou M (2010) Biodegradation, biodistribution and toxicity of chitosan. Adv

336 Drug Deliv Rev 62:3-11

337 Lavertu M, Méthot S, Tran-Khanh N, Buschmann MD (2006) High efficiency gene

338 transfer using chitosan/DNA nanoparticles with specific combinations of molecular

339 weight and degree of deacetylation. Biomaterials 27:4815-4824

340 Mao HQ, Roy K, Troung-Le VL, Janes KA, Lin KY, Wang Y, August JT, Leong KW

341 (2001) Chitosan-DNA nanoparticles as gene carriers: synthesis, characterization and

342 transfection efficiency. J Control Release 70:399-421

343 Mao S, Sun W, Kissel T (2010) Chitosan-based formulations for delivery of DNA and

$344 \quad$ siRNA. Adv Drug Deliv Rev 62:12-27

345 Motohashi R, Shimojima T, Fukagawa T, Maenaka K, Park EY (2005) Efficient

346 large-scale protein production and pupae of slarvae and pupae of silkworm by 
347 Bombyx mori nuclear polyhedrosis virus bacmid system. Biochem Biophys Res

$348 \quad$ Commun 326:564-569

349 Ogata M, Nakajima M, Kato T, Obara T, Yagi H, Kato K, Usui T, Park EY (2009)

350 Synthesis of sialoglycopolymerpeptide for potentially blocking influenza virus

351 infection using a rat alpha2,6-sialyltransferase expressed in BmNPV

352 bacmid-injected silkworm larvae. BMC Biotechnol 9:54

353 Otsuki T, Dong J, Kato T, Park EY (2013) Expression, purification and antigenicity of

354 Neospora caninum-antigens using silkworm larvae targeting for subunit vaccines.

$355 \quad$ Vet Parasitol 192:284-287

356 Park EY, Kageshima A, Kwon MS, Kato T (2007) Enhanced production of secretory

357 beta1,3-N-acetylglucosaminyltransferase 2 fusion protein into hemolymph of

358 Bombyx mori larvae using recombinant BmNPV bacmid integrated signal sequence.

$359 \quad$ J Biotechnol 129:681-688

360 Park EY, Abe T, Kato T (2008) Improved expression of fusion protein using a

361 cysteine-protease and chitinase-deficient Bombyx mori (silkworm) multiple

362 nucleopolyhedrovirus bacmid in silkworm larvae. Biotechnol App Biochem 49:135-

$363 \quad 140$

364 Rojanarata T, Opanasopit P, Techaarpornkul S, Ngawhirunpat T, Ruktanonchai U

365 (2012) Chitosan-thyamine purophosphate as a novel carrier for sirna delivery.

366 Pharm Res 25:2807-2814

367 Romøren K, Pedersen S, Smistad G, Evensen Ø, Thu BJ (2003) The influence of

368 formulation variables on in vitro transfection efficiency and physicochemical

369 properties of chitosan-based polyplexes. Int J Pharm 261:115-127 
370 Sato T, Ishi T, Okahata Y (2001) In vitro gene delivery mediated by chitosan. Effect of

$371 \mathrm{pH}$, serum, and molecular mass of chitosan on the transfection efficiency.

$372 \quad$ Biomaterials 22:2075-2080

373 Steg AD, Karte AA, Goodman BW, Han HD, Nick AM, Stone RL, Coleman RE,

374 Alvarez RD, Lopez-Berestein G, Sood AK, Landen AK, Landen SN (2011)

375 Targeting the Notch ligand jagged1 in both tumor cells and stroma in ovarian cancer.

$376 \quad$ Clin Cancer Res 17:5674-5685

377 Tripathi SK, Goyal R, Kumar P, Gupta KC (2012) Linear polyethylenimine-graft

378 chitosan copolymers, as efficient DNA/siRNA delivery vectors in vitro and in vivo.

$379 \quad$ Nanomedicine 8:337-345

380 


\section{Figure legends}

382 Fig. 1 Particle size and zeta potential of chitosan/BmNPV bacmid DNA

383 nanocomplexes in the N/P ratio range of $0.1-10$. These particles were prepared using

384 Chitosan 100 and recombinant BmNPV bacmid harboring GGT2 gene at each N/P ratio.

385 Particle size and zeta potential of these particles were analyzed dynamic light scattering

386 (DLS) analysis.

387 Fig. 2 Electrophoretic mobility analysis of chitosan/BmNPV bacmid DNA

388 nanocomplexes. A Agarose gel electrophoresis of chitosan/BmNPV bacmid DNA

389 nanocomplexes of various N/P ratios of 0.1-10. B Agarose gel electrophoresis of

390 DNase-treated chitosan/BmNPV bacmid DNA nanocomplexes of various N/P ratios of

391 0.5-10. (+) and (-) denote chitosan/BmNPV bacmid DNA nanocomplexes treated with

392 DNase and without, respectively.

393 Fig. 3 Expression of GGT2 in Bm5 cells using chitosan/BmNPV bacmid DNA

394 nanocomplexes. A Fluorescent microscopy of Bm5 cells transfected with

395 chitosan/BmNPV bacmid DNA nanocomplexes. Chitosan 100 and recombinant

396 BmNPV bacmid harboring GGT2 gene at each N/P ratio were used for

397 chitosan/BmNPV bacmid DNA nanocomplexes. After $7 \mathrm{~d}$ incubation, green

398 fluorescence in Bm5 cells was detected by confocal fluorescence microscope. B

399 Expression of GGT2 in Bm5 cells transfected with chitosan/BmNPV bacmid DNA

400 nanocomplexes prepared at N/P ration 4 or 6 . Used amount of recombinant bacmid

401 DNA was 2.5, 5, and $10 \mu \mathrm{g}$. Green fluorescent bands of GGT2 on an SDS-PAGE gel

402 were detected by Molecular imager FX. 
403 Fig. 4 Expression of GGT2 in silkworm larvae using chitosan/BmNPV bacmid DNA

404 nanocomplexes. A Green fluorescent of DMRIE-C- and chitosan/BmNPV bacmid DNA

405 nanocomplexes-injected 10 silkworm larvae with under UV light. B Expression of

406 GGT2 in silkworm larvae injected with chitosan/BmNPV bacmid DNA nanocomplexes

407 prepared at each N/P ration using $5 \mu \mathrm{g}$ of recombinant bacmid DNA. Green fluorescent

408 bands of GGT2 in hemolymph on an SDS-PAGE gel were detected by Molecular

409 imager FX.

410 Fig. 5 Specific $\beta 3 \mathrm{GnT}$ activity of hemolymph in silkworm larvae using

411 chitosan/BmNPV bacmid DNA nanocomplexes at each N/P ratio. Five microgram of

412 recombinant BmNPV bacmid was used for protein expression in silkworm larva.

$413 \quad \beta 3 \mathrm{GnT}$ assay and protein concentration measurement are described in Materials and

414 methods $(\mathrm{n}=3)$. Error bars indicate standard deviation. Student's t-test was performed to

415 find significant difference between two means $(p<0.05)$.

416 Fig. 6 Expression of rat ST6, HA from influenza A H5N8 virus and NcSRS2 from $N$.

417 caninum in chitosan/BmNPV bacmid DNA nanocomplexes-injected silkworm larval

418 hemolymph. Chitosan 100 and each recombinant bacmid DNA were used at N/P ratio 5

419 for the preparation of chitosan/bacmid DNA nanocomplexes. Five microgram of

420 recombinant BmNPV bacmid was used for protein expression in silkworm larva. Lane1:

421 Mock, lane 2: DMRIE-C-BmNPV bacmid DNA, lane 3: Chitosan/BmNPV bacmid

422 DNA nanocomplex. 
Fig. 1 Kato et al.

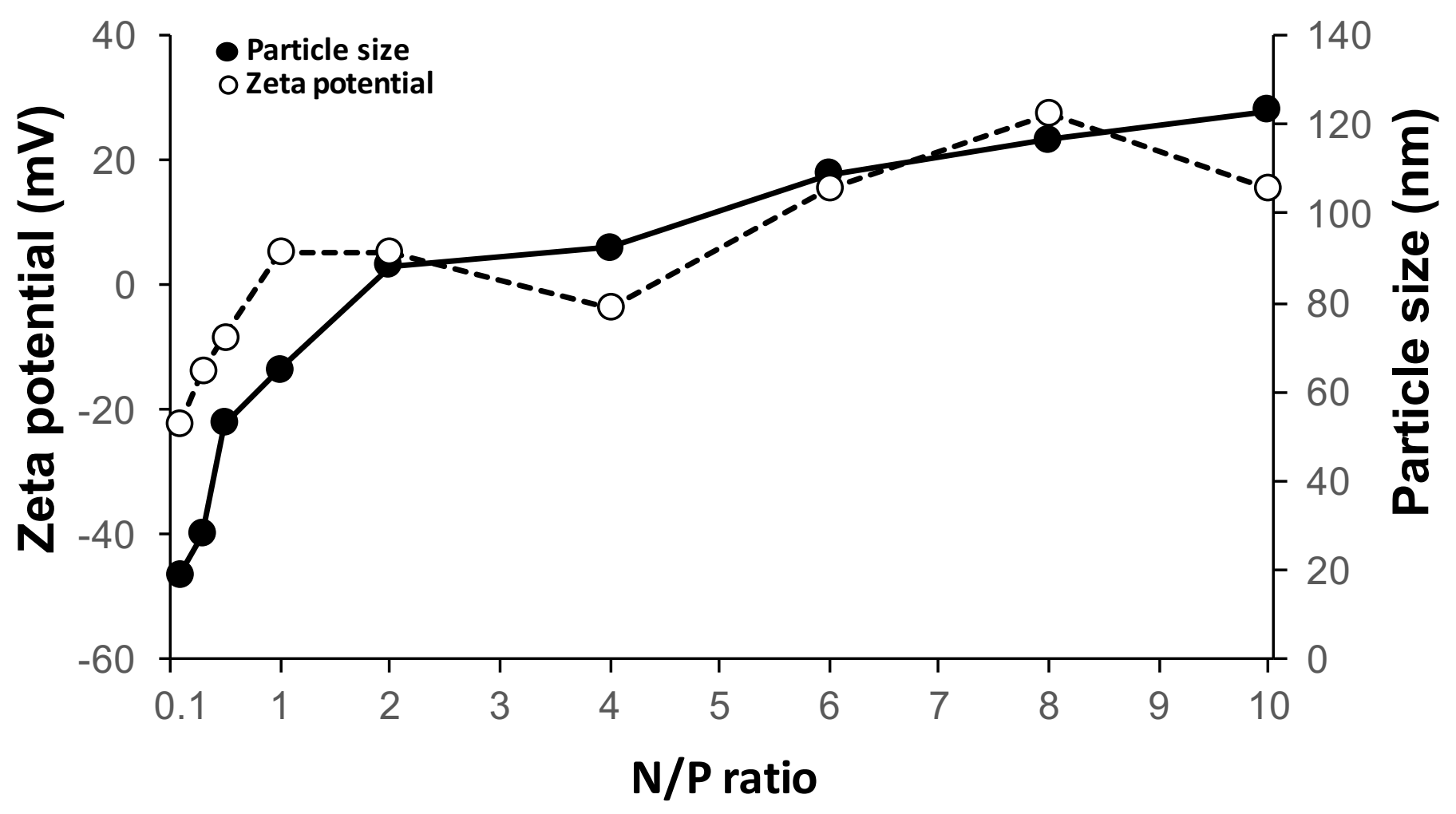


A: DNase -

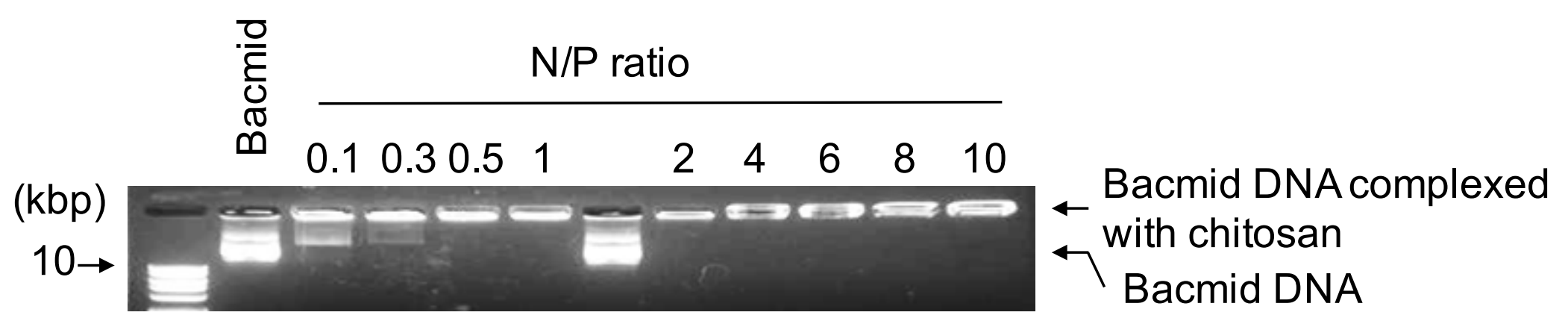

B: DNase +

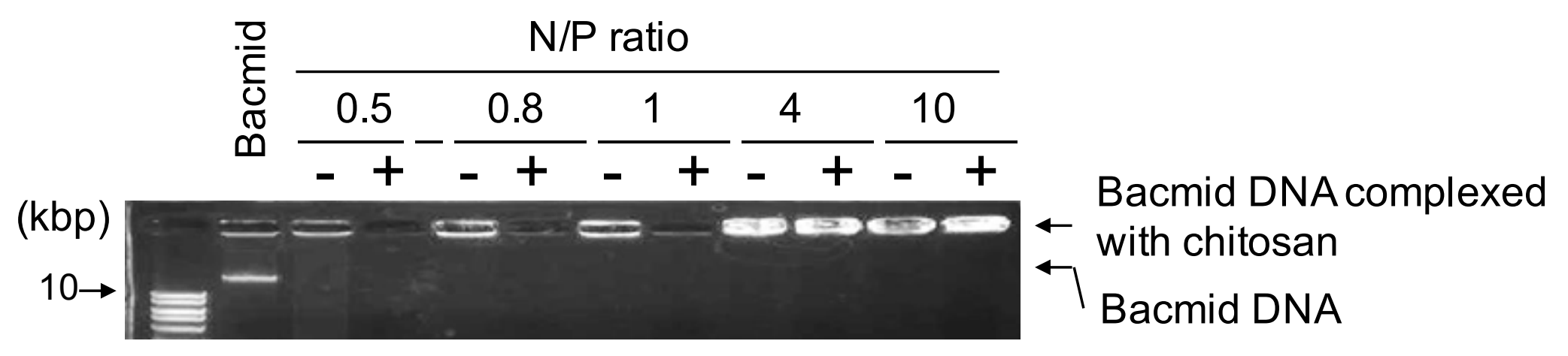


Fig. 3 Kato et al.

A
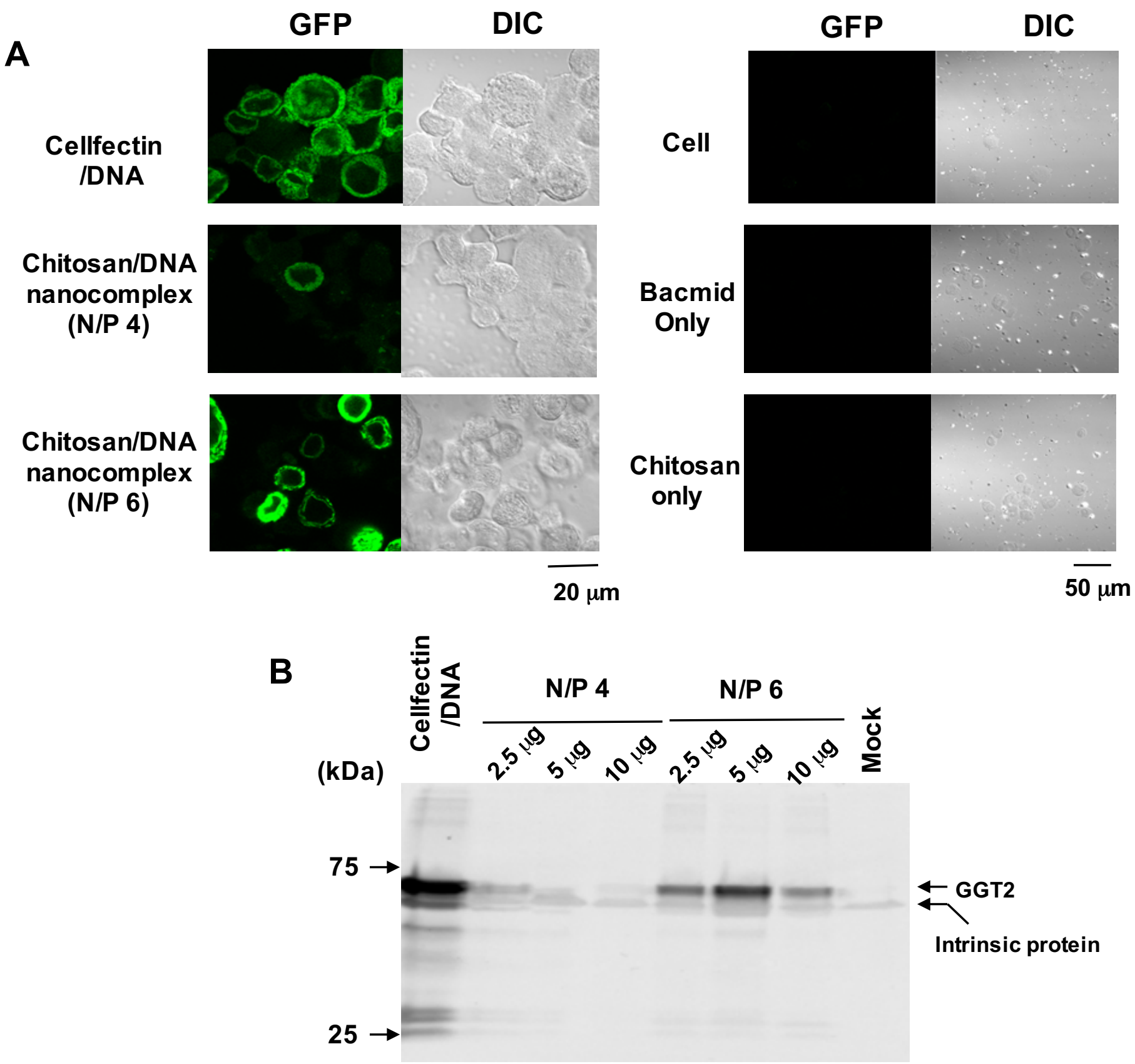
Fig. 4 Kato et al.
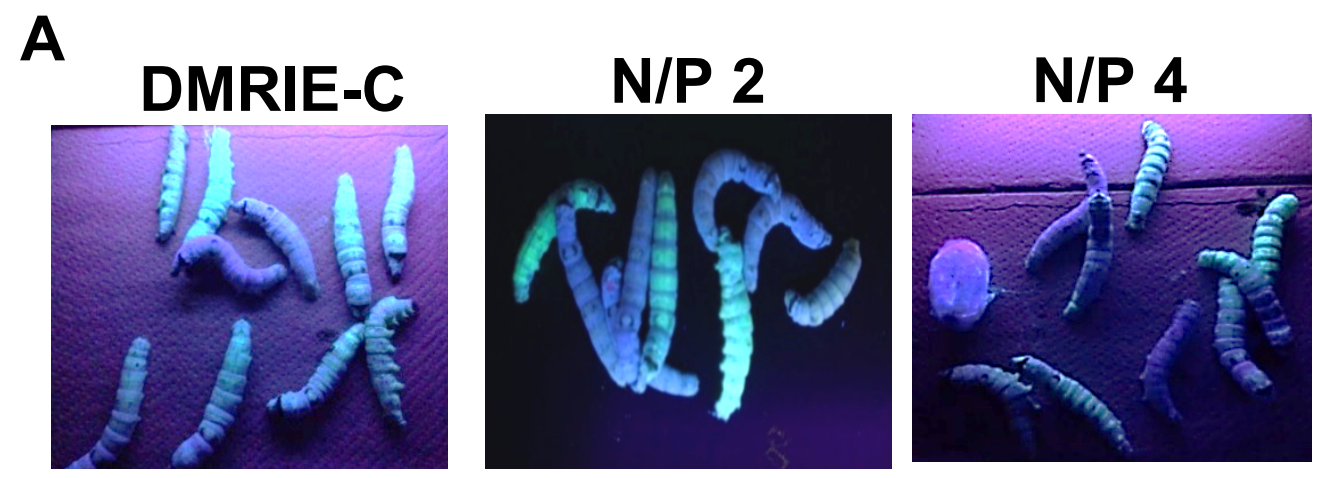

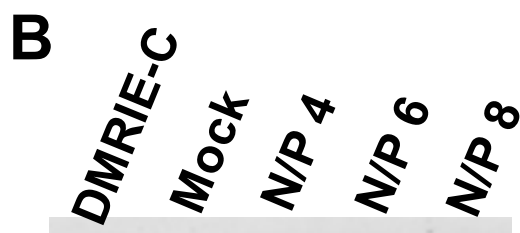

N/P 6

N/P 8
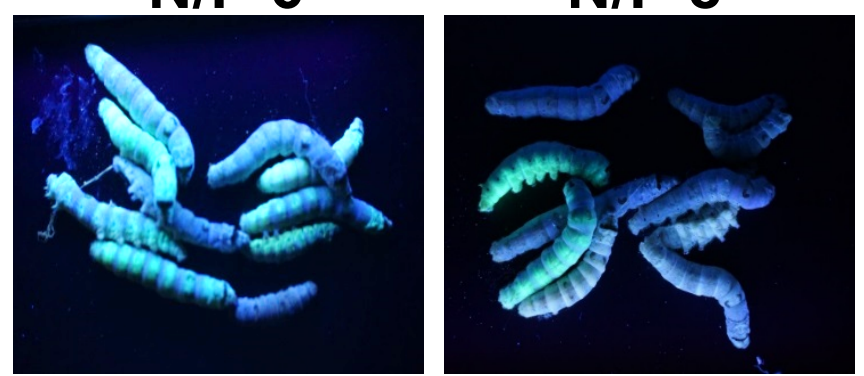

$\leftarrow$ GGT2 


\section{Fig. 5 Kato et al.}

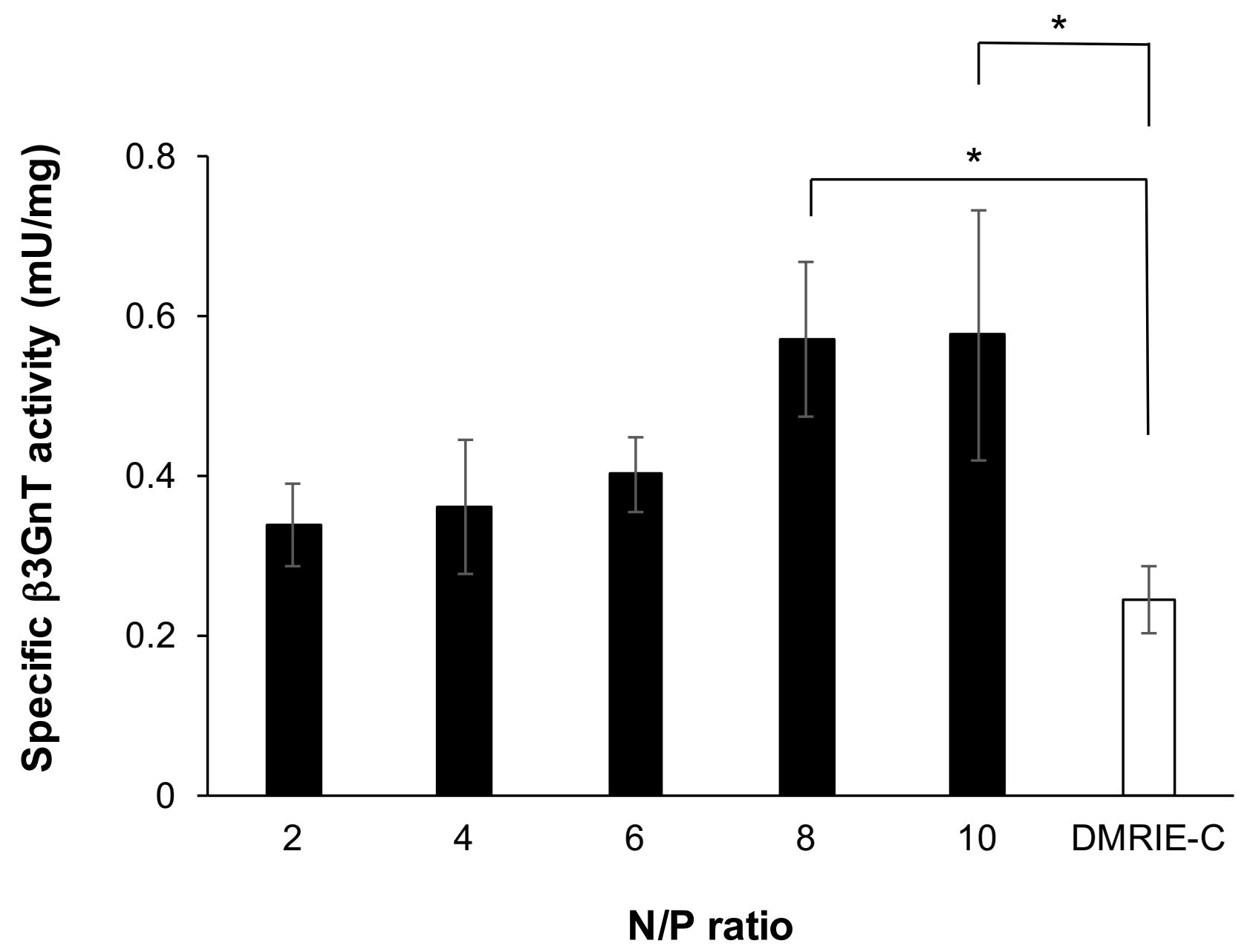


Rat ST6

123

$-\infty \leftarrow 36 \mathrm{kDa}$

\section{HA}

123

\section{NcSRS2}

123
의 $\leftarrow 50 \mathrm{kDa}$
$\leftarrow 40 \mathrm{kDa}$ 